\title{
Rapid neuronet inversion of 2D magnetotelluric data for monitoring of geoelectrical section parameters
}

\author{
Mikhail I. Shimelevich $\left({ }^{1}\right)$, Eugeny A. Obornev $\left({ }^{1}\right)$ and Sergei Gavryushov $\left({ }^{2}\right)$ \\ (1) Moscow State Geoprospecting University, Moscow, Russia \\ ${ }_{\left({ }^{2}\right)}$ Engelhardt Institute of Molecular Biology, Moscow, Russia
}

\begin{abstract}
The inverse MagnetoTelluric (MT) operator is approximated by means of the Neural Network (NN). The methodology of the NN interpretation in classes of the geoelectrical sections described by the hundreds of parameters is proposed. Error of the NN inversion and field misfit are evaluated. A rapid NN algorithm solving the inverse problem and detecting changes of time-dependent dynamic parameters of the section is applied to 2D synthetic data.
\end{abstract}

Key words magnetotelluric inverse problem - neural network - EM monitoring

\section{Introduction}

In recent years some examples of the Neural Network (NN) technique applications for solution of the magnetotelluric inverse problems (Poulton, 2002) and rapid inverse problems (Zhdanov and Chernyavskiy, 2004) have been presented. It was shown that this method is effective when the number of the geoelectrical section parameters is about 10 (Spichak and Popova, 2000; Spichak et al., 2002; Shimelevich et al., 2002), i.e. the solution is sought within a narrow class of models. An important peculiarity of almost instant $\mathrm{NN}$ inversion makes this approach attractive for the real-time monitoring of electromagnetic parameters of the medium. It should be added that the $\mathrm{NN}$ can

Mailing address: Dr. Mikhail I. Shimelevich, Moscow State Geoprospecting University, 123001, Sadovaya Kudrinskaya str., 22, building 1, Moscow, Russia; e-mail: shimelevich@ecc.ru be efficiently applied to the monitoring of a few parameters of the known section using a rarefied set of measurements (Shimelevich et al., 2003). In the present paper the NN technique is shown to be effective to reconstruct the conductivity distribution and its time variations in case of hundreds of parameters within a wide class of geoelectrical models.

\section{Metodology of the $\mathrm{NN}$ inversion in a class of the geoelectrical sections}

The inverse MT problem of the evaluation of the vector of geoelectrical section parameters $\gamma=\left(\gamma_{1}, \ldots, \gamma_{N}\right)$ according to the MT data $\beta=$ $=\left(\beta_{1}, \ldots, \beta_{M}\right)$ observed on the Earth surface can be reduced to a solution of the non-linear operator equation $A_{k} \gamma=\beta, \gamma \in \Gamma_{k}$; where $\Gamma_{k}$ its set associated with the given class $G_{k}, k$ is the class number and $A_{k}$ is the MT forward problem operator, defined on the subset $\Gamma_{k}$.

The NN approach is an approximation of the inverse operator $S_{k}=A_{k}^{-1}$ by a superposition of non-linear functions of the given type. One of the widespread NN approximations is the multilayer perceptron (Raiche, 1991). In this 
case, a solution of the inverse problem is sought as: $\gamma=f(V f(W \beta))$, where the sigmoid functions are $f(x)=1 /(1+\exp (-x))$. To calculate the unknown matrices $V, W$ the method of NN learning with a teacher is used. The NN is learned, using a database of samples that are pairs of vectors $\left\{\gamma_{p}, \beta_{p}\right\}$, obeying the equation: $A_{k} \gamma_{p}=\beta_{p}$, $\gamma_{p} \in \Gamma_{k}, p=1, \ldots, P$ ( $P$ is a number of samples). Vector $\gamma_{p}$ is randomly chosen from the set $\Gamma_{k}$. A numerical implementation of the method based on the error Back Propagation algorithm (Raiche, 1991). To estimate its interpolation properties and quality of the inversion, the independent testing set is used. There are calculated parameter errors $\Delta_{k}=\left(\Delta_{k_{1}}, \ldots, \Delta_{k_{N}}\right)$ and the field misfit $\delta_{k}$ averaged for the testing set. This independent set of samples is used to verify the accuracy of the inversion. This accuracy is increased at growth of the number of samples $P$ (Shimelevich et al., 2001). To obtain a practically applicable accuracy of the inversion (about a few percent), the database can include up to hundreds of thousand of examples. Parallel algorithms are used to obtain such a database.

Learned neuronet $S_{k}$ determines an approximate inverse operator $A_{k}{ }^{-1}$ for the model class $G_{k}$ of the geoelectrical sections, the parameter errors $\Delta_{k}=\left(\Delta_{k_{1}}, \ldots, \Delta_{k_{N}}\right)$ and the field misfit $\delta_{k}$ obtained as the result of the NN testing allow us to estimate the error of the interpretation, and to solve a problem of a correspondence between measured data and medium classes $G_{k}$ (Shimelevich and Obornev, 1999). To evaluate the impact of the noise, learned neural networks were tested for data with added noise of various kinds and magnitudes. Results suggested that relative errors of the inversion led to field deviation not exceeding the norm of the noise (Shimelevich et al., 2001).

\section{The classes of the parameterized geoelectrical sections}

In practice, an interpreter can deal with several hypotheses on the conductivity distribution model, so the solution of the inverse problem should be sought in a few classes $G_{k}$ of the medium. In this case, it is necessary to have a set of neuronets $S_{0}, S_{1}, \ldots$, corresponding to dif-

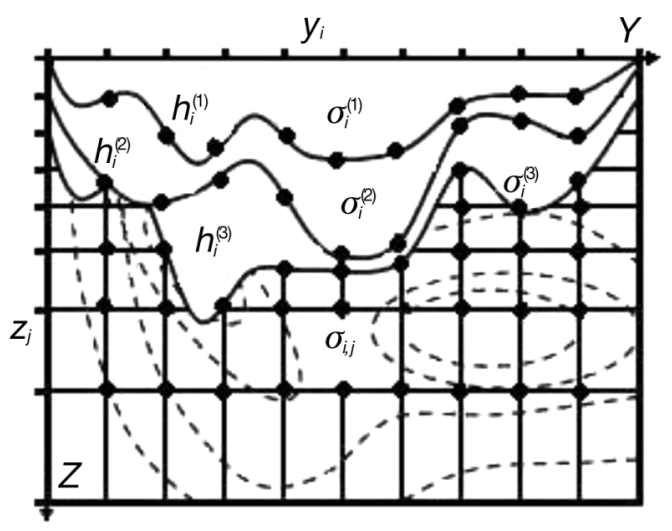

Fig. 1. The generalised example of the geoelectrical section parameterization. The model can include $N$ layers (1), (2), (3) and 2D conductivity grid. The conductivities at the nodes of the 2D grid, thicknesses and conductivities of the layers in the horizontal direction are varied.

ferent classes $G_{0}, G_{1}, \ldots$ of the parameterized geoelectrical sections. The parameterized class $G_{k}$ is the set of all possible geoelectrical sections which conductivity can be described as: $\sigma(y, z)=f_{k}\left(y, z, \gamma_{1}, \ldots, \gamma_{N}\right), y, z \in \Omega, \gamma_{n}^{\text {min }} \leq \gamma_{n} \leq$ $\leq \gamma_{n}^{\max }, n=1, \ldots, N_{k}$. Here $\Omega$ is a domain of $R^{2}$, where the electromagnetic problem is formulated; $\gamma_{n}^{\min }, \gamma_{n}{ }^{\max }$ are the limits of the $\gamma_{n}$ parameter variation, the $f_{k}$ is the function of the parameterization of the given class and $N_{k}$ is the number of parameters which depends on the complexity of the class $G_{k}$.

The generalized example of the section parameterization is shown in fig. 1 . The parameters of the structure are the layer thicknesses defined at the fixed nodes, the conductivities of the blocks within the layers and the conductivities at the fixed nodes of the rectangular grid. For the forward problem solving the parameterized conductivity of the medium is interpolated between the nodes. The number of sought parameters of such structures is about a few hundreds, whereas the finite-difference scheme dimension of the corresponding boundary value problem is of tens of thousands.

We will consider three particular examples of medium classes. 
Class $\boldsymbol{G}_{\mathbf{0}}(\mathrm{Grid})$ - It is the most general class of the geoelectrical sections. The conductivity can change within a fixed range at all nodes of the 2D grid. Between the nodes the conductivity is interpolated by a 2D spline.

Class $\boldsymbol{G}_{\mathbf{1}}$ (Layer+Grid) - The upper layer of the section is described explicitly, whereas the rest of the section is parameterized as in class $G_{0}$. The layer thickness varies within a given range at the fixed nodes in the horizontal direction. Between the nodes the layer boundaries are interpolated by a 1D spline. The layer conductivity varies linearly between the centers of the blocks in the horizontal direction.

Class $\boldsymbol{G}_{\mathbf{2}}$ (3 layers+Grid) - The upper three layers of the section are described explicitly. The layer boundaries are parameterized as in class $G_{1}$. The layer conductivity varies linearly between the centers of the blocks in the horizontal direction. A total number of parameters of the de- scribed conductivity classes is from 233 to 336. For these classes neuronets $S_{0}, S_{1}, S_{2}$ were learned according to the described above principles.

\section{NN interpretation of the $2 \mathrm{D}$ synthetic data}

The structure used to generate the synthetic data is shown in fig. 2a. This structure (Model 1) includes three layers of the changeable thickness and the underlying stratum. The thickness of the upper layer varies from 100 to $3900 \mathrm{~m}$. Its conductivity varies in the horizontal direction from 0.185 to $0.008 \mathrm{~S} / \mathrm{m}$. The conductivities of the second, third layers and of the underlying half-space are constant and equal $\sigma_{2}=$ $=0.0004 \mathrm{~S} / \mathrm{m}, \sigma_{3}=0.2 \mathrm{~S} / \mathrm{m}, \sigma_{4}=0.0002 \mathrm{~S} / \mathrm{m}$. The thickness of the second and third layers vary from 0 to $13.4 \mathrm{~km}$ and from 2 to $4.8 \mathrm{~km}$. The apparent resistivities and phases for TE and
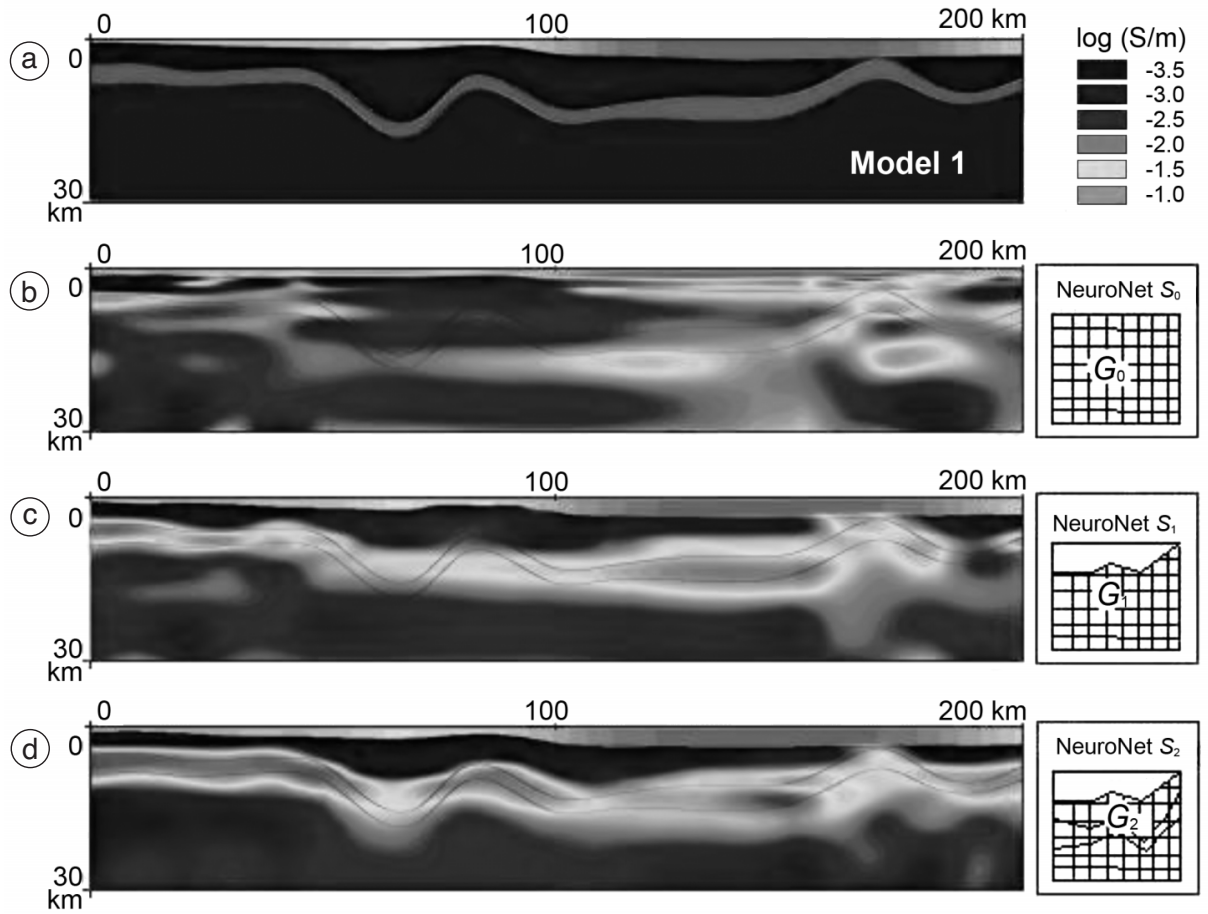

Fig. 2a-d. The results of the synthetic data interpretation. a) Model 1 used to generate the synthetic data; b) interpretation, step1 (NN $S_{0}$ is applied); c) interpretation, step2 (NN $S_{1}$ is applied); d) interpretation, step3 (NN $S_{2}$ is applied). The thin solid lines are true boundaries of the layers of Model 1. 
TM modes were calculated at 13 periods from 0.01 to $1000 \mathrm{~s}$ in 126 sites along the profile.

To interpret the generated synthetic data a set of learned neuronets $S=\left(S_{0}, S_{1}, S_{2}\right)$ was used. At the first stage of the interpretation there is no hypothesis about the model class of the section. So, we have to use neuronet $S_{0}$ corresponding to the widest class $G_{0}$ of geoelectrical sections. The result of the first inversion (step 1) is shown in fig. 2b. At this stage of the interpretation, one can draw the only conclusion that there might be a thin superficial conductive layer. As is seen from averaged field misfits given in fig. 3, all results of imaging related to the deeper part of the section are unreliable on this first step of inversion. A relatively low misfit can be observed only at high frequencies, which suggest that one can only trust conductivity distribution close to the surface. Taking this conclusion as a starting point, one can use neuronet $S_{1}$ corresponding to the class (Layer+Grid). The result of the second inversion (step 2) is shown in fig. 2c. The upper lay-

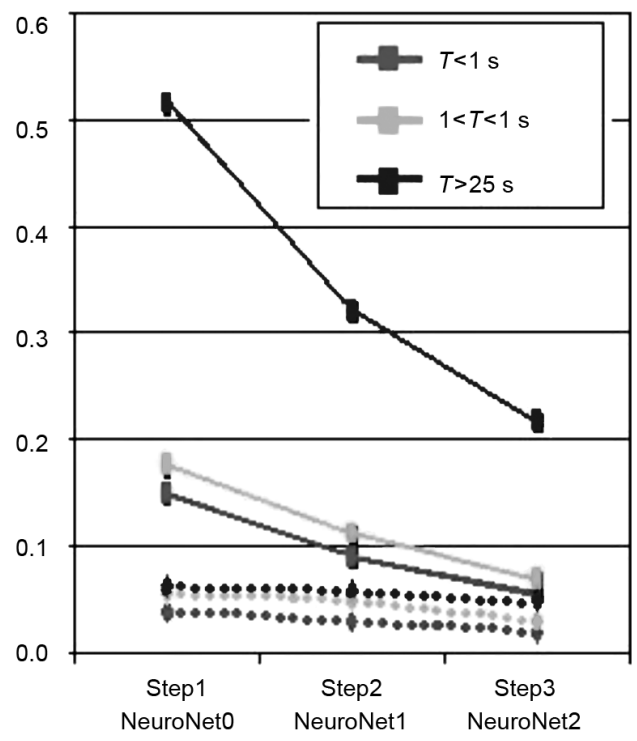

Fig. 3. Field misfits averaged over three period ranges: $T<1 \mathrm{~s} ; 1<T<25 \mathrm{~s} ; T>25 \mathrm{~s}$ at the three $\mathrm{NN}$ inversions (solid lines). Dotted lines are averaged field misfits obtained at the neuronet testing. er is seen better and its lower boundary is practically identical to the true boundary. Three layers can be seen under it and the conductivity of the third layer is about $0.1 \mathrm{~S} / \mathrm{m}$. At the same time the field misfit has decreased in the whole frequency range (see fig. 3). This proves the correctness of using neuronet $S_{1}$ on step 2. Taking into account this fact and the image of the section in fig. 2c one can try to use neuronet $S_{2}$ corresponding to the class $G_{2}$ (3 layers+Grid) at the next stage of the interpretation. The result of the inversion (step 3) is shown in fig. $2 \mathrm{~d}$. The second conductive layer is seen better and the field misfit at the range $T \leq 25$ has dropped to the level of the mean field misfits obtained at the testing of neuronet $S_{2}$. Thus, one can see that the inversion with using neuronet $S_{2}$ gives a satisfactory variant of the interpretation except for the deepest part of the section.

An application of the $\mathrm{NN}$ requires knowledge of the model class of the resistivity section. If there is no such information, an interpreter has to invert data using NN $S_{0}, S_{1}$, which justifies the final application of the NN $S_{2}$. It is important to note that application of $S_{0}$ does not required any information since it is based on the most general class $G_{0}$. The sequence of inversions $S_{0}, S_{1}$ illustrates an application of the NN at the lack of $a$ priori information about the section.

\section{Rapid detection of the geoelectrical parameter variations}

To estimate the resolving power of the NN inverse operator the boundary of the third layer of Model 1 was changed in three zones marked by the dashed line in fig. 4. After forward modeling for the modified section, the new synthetic MT data were interpreted using neuronet $S_{2}$. The difference between the results of the interpretations is shown in fig. 4. As one can see the difference conductivity map localizes the zones of the conductivities changes with a good accuracy. Note, that we can apply the same learned neuronet for inversion, since the modified section belongs to the same model class as the initial section. In this case the data interpretation can be done very quickly (several seconds on a single computer). This example shows a high 


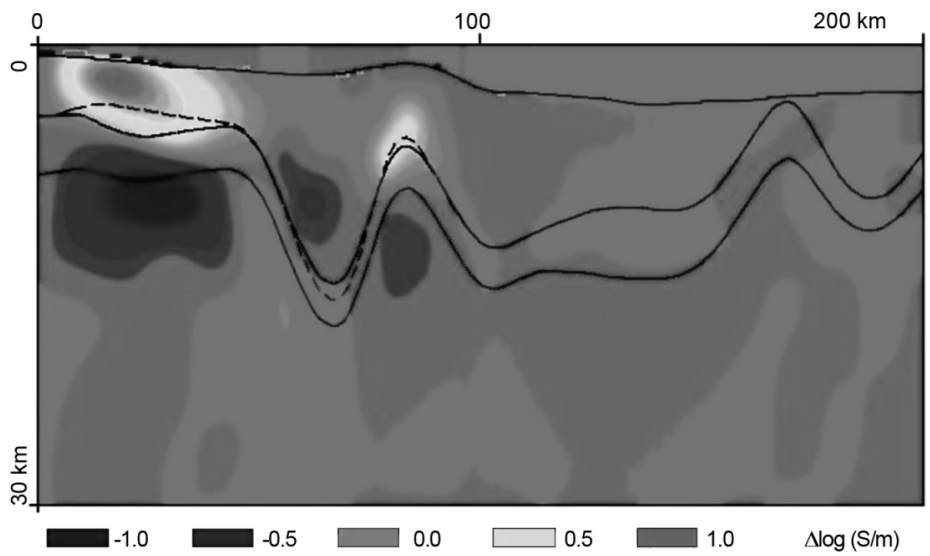

Fig. 4. The difference between the results of the interpretation of the initial and modified geoelectrical sections. The changed boundaries are marked by the dashed line. The solid lines are the boundaries of Model 1.

sensitivity of the NN inverse operators to the geoelectrical parameter variations. It illustrates a possibility of a real-time monitoring of resistivity section parameters if these changes can be detected from the measured fields.

\section{Conclusions}

1) A representative set of the learned neuronets allows us to interpret a $2 \mathrm{D}$ geoelectrical sections described by hundreds of parameters and to estimate the accuracy of the interpretation results.

2) Due to rapidity of NN inversion and high resolution the neuronet technology could be used for the monitoring and localization of small variations of the conductivity distribution in a real time scale.

A clear disadvantage of the method is a large number of the trained $\mathrm{NN}$ for different classes of the geoelectrical sections and difficulty to choose a true NN at the lack of information.

\section{REFERENCES}

Poulton, M.M. (2002): Neural networks as an intelligence amplification tool: a review of applications, Geophysics, 67 (3), 979-993
RAICHE, A. (1991): A pattern recognition approach to geophysical inversion using neural nets, Geophys. J. Int., 105, 629-648.

ShimeleVICH, M.I. and E.A. OBoRnEV (1999): The method of neural network applied to the approximation of the inverse operators in electromagnetic sounding problems, Izvestiya Vuzov (Geol. Prospect.), 2, 102-106 (in Russian).

ShimeleVICH, M.I., E.A. ObORNEV and S.A. GAVRYUShov (2001): A method of designing neural networks for solving multiparametric inverse problems of magnetotelluric sounding, Izvestiya Vuzov (Geol. Prospect.), 6, 129-137 (in Russian).

Shimelevich, M.I., E.A. OBornev and S.A. GAVRYushov (2002): Neuronet approximation of the inverse MT operators for geoelectrical monitoring, in Proceedings of III International Workshop on Magnetic, Electric and Electromagnetic Methods in Seismology and Volcanlogy (MEEMSV-2002), September 3-6, Moscow, 59-62.

ShimeleVICH, M.I., E.A. OborneV and S.A. GaVRYushov (2003): Application of neuronet approximation to the solution of geo-electromonitoring problems, Izvestiya Vuzov (Geol. Prospect.), 4, 70-71 (in Russian).

SPICHAK, V. and I. POPOVA (2000): Artificial neural network inversion of magnetotelluric data in terms of three-dimensional Earth macroparameters, Geophys. J. Int., 142, 15-26.

Spichak, V., K. Fukuoka, T. Kabayashi, T. Mogi, I. PopoVA and H. SHIMA (2002): ANN reconstruction of geoelectrical parameters of the Mionou fault zone by scalar CSAMT data, J. Appl. Geophys., 49, 75-90.

Zhdanov, M.S. and A. ChernyavskiY (2004): Rapid threedimensional inversion of multi-transmitter electromagnetic data using the spectral Lanczos decomposition method, Inverse Problems, 20, S233-S256. 